\title{
Immune Response in the Common Raccoon (Procyon lotor) to Modified Live Canine Distemper and Feline Panleukopenia Vaccines
}

\author{
Anne Staudenmaier ${ }^{1}$, Erica Miller, DVM ${ }^{1}$, and Joseph Dubovi, MA, PhD ${ }^{2}$ \\ ${ }^{1}$ College of Veterinary Medicine, University of Pennsylvania, Philadelphia, Pennsylvania. \\ ${ }^{2}$ College of Veterinary Medicine, Cornell University, Ithaca, New York
}

\begin{abstract}
The common raccoon, Procyon lotor, while in a separate family from the dog and cat, is susceptible to diseases traditionally associated with these domestic small animals. Thus, the species' potential for transmitting these devastating diseases back to these small domestic animals should not be ignored. Though vaccinating wild raccoons for canine distemper and feline panleukopenia has been a common practice in wildlife rehabilitation for the past few decades, little is known about the actual efficacy of these vaccines. The purpose of this study was to investigate the antibody titers produced by a commonly used vaccination protocol in wild raccoons for canine distemper and the parvoviruses. Pre- and post-vaccination blood was taken from 49 orphaned raccoons brought to Mercer County Wildlife Center in Titusville, New Jersey, and samples were sent to Cornell University for titer determination. Animals with low pre-vaccination antibody levels showed appreciable rises in titers for canine distemper. For the parvovirus family, while still protective in most cases, titer rise was much less dramatic, and in two cases, vaccine failure was noted. Raccoons presenting with positive titers pre-vaccination-most likely due to maternal colostral protection-did not develop a significant immunological response to vaccination if titers were above 1:192, suggesting that colostral antibodies may persist long enough to interfere with current vaccination schedules used by rehabilitation centers and protocols may need to be adjusted to extend for longer time periods.
\end{abstract}

Keywords: Canine distemper, panleukopenia, parvovirus, Procyon lotor, vaccine-induced immunity

\section{INTRODUCTION}

Canine distemper and feline panleukopenia viruses represent important infectious disease risks to many different species worldwide. The viruses continue to threaten a vast array of animals across the globe, and the threat to populations of endangered wildlife and unvaccinated domestic animals remains a constant problem in many countries.

Anne Staudenmaier Anne Staudenmaier is a fourth-year student currently completing her veterinary doctorate at the University of Pennsylvania School of Veterinary Medicine.

Erica Miller, is a wildlife veterinarian with the New Jersey Division of Fish \& Wildlife. She is a past president and former board member of NWRA.

Joseph Dubovi, MA, PhD is the Director of the virology laboratory at Cornell University Animal Health Diagnostic Center and professor of virology at Cornell University College of Veterinary Medicine. He has authored numerous journal articles relating to wildlife medicine.
Evidence of canine distemper infection has been reported worldwide in all terrestrial carnivore families, as well as in non-human primates, suids, pinnipeds, and porpoises (Deem et al 2000; Durchfeld et al 1990; Van Moll et al 1995; Wimsatt et al 2006). Other than rabies, canine distemper remains the infectious disease with the highest fatality rate in animals (Appel et al 1995). For endangered carnivore species, it is the most significant viral pathogen known, devastating populations of lions (Panthera leo) (Roelke-Parker et al 1996), snow leopards (Panthera uncia), red pandas (less/lesser panda) (Ailurus fulgens) (Chappius 1995; Wimsatt et al 2006), Caspian seals (Pusa caspica) (Kennedy et al 2000), and African wild dogs (painted dog, Cape hunting dog, Lycaon pictus) (Durchfeld et al 1990). In addition, this disease has been implicated in the extinction of the Tasmanian tiger (Thylacinus cynocephalus) and the near-extinction of the black-footed ferret (Mustela nigripes) (Deem et al 2000; Paddle 2014). The parvovirus family is composed of antigenically-related diseases, including canine parvovirus, raccoon parvovirus, and mink enteritis virus, and all are believed to have derived from feline panleukopenia (Parrish and Carmichael 1983; Parrish et al 1988; Parrish 1995; Tratschin et al 1982). Despite the viruses being named for the host from which they were first isolated, studies have demonstrated many species can be infected with multiple members of tshis viral family and interspecies transmission can and does occur (Mochizuki et al 1996). Parvoviridae has had serious, negative impacts on members of the mustelidae family, along with felids and procyonids (Durate et al 2013; Parrish and Carmichael 1983; Truyen and Parrish 1992).

Of growing concern, recent studies have suggested there may be a relationship between these diseases and multiple sclerosis, Paget's disease, and subacute sclerosing panencephalitis (Deem et al 2000). It has been shown experimentally that the canine distemper virus 
can infect human cells, and while no definitive evidence exists to show that natural infections of humans by these diseases has occurred, their threat as possible zoonoses cannot be dismissed (Deem et al 2000).

In North America, the common raccoon remains highly susceptible to both canine distemper and multiple parvoviruses. Mortality in naïve populations of animals can reach 50 to 90 percent of populations for canine distemper and as high as 100 percent for feline panleukopenia (Barker et al 1983). Juveniles that have just lost the protection of maternal antibodies are most at risk for infection, shedding, and disease (Appel et al 1995). In addition, and of significant public health concern, raccoons can perpetuate the life cycles of distemper and parvovirus within the environment. Since they often are unable to mount an effective antibody response to infection, carrier states are common (Bittle 1993). Raccoons have been implicated in multiple outbreaks of canine distemper virus and parvovirus among lions, snow leopards, tigers, black-footed ferrets, and mink (Mustelidae sp.) (Appel et al 1994; Durate et al 2013; Fix et al 1989; Oie et al 1996). Raccoons serve as reservoirs for these diseases (Van Moll et al 1995) and can spread infection to unvaccinated domestic animals in both urban and rural settings.

Experts agree that a control program for these diseases requires vaccination at its core, but vaccination strategies in reality continue to be problematic. Killed vaccines have proven to be ineffective in providing protection, and modified-live vaccines, especially those for canine distemper, have been associated with adverse reactions. Canine distemper vaccines can cause immunosuppression and even rare reversion to virulence in dogs (Chappius 1995), and fatal vaccineinduced distemper infections have been reported in the black-footed ferret, red panda, African wild dog, and kinkajou (Potos flavus) (Appel 1994; Durchfeld et al 1990; Woodroffe 1999). Because of the sensitivity of exotic and wildlife species to modified-live vaccines, the vaccination of wild animals that can serve as reservoirs has been advocated as an alternative.

In response to the implications of infected procyonids, many wildlife rehabilitation centers vaccinate orphaned juvenile raccoons for both canine distemper and feline panleukopenia, yet little information exists on the relative efficacy of these vaccines in raccoons. Relatively few studies have examined the change in antibody levels in the raccoon following vaccination (Pare et al 1999, for distemper, and none for the feline and mink parvoviruses). This study aimed to investigate the efficacy of a current vaccination protocol in wild raccoons for both canine distemper and the parvoviruses.

\section{MATERIALS AND METHODS}

Animal and Sample Collection. Forty-nine orphaned juvenile raccoons from nine New Jersey (NJ) counties were brought in to the Mercer County Wildlife Center in Titusville, NJ between May and August 2013. Animals were anesthetized using isoflurane upon entry and physical exams were performed. Pre-vaccination blood samples between $0.5 \mathrm{cc}$ and 2.0 $\mathrm{cc}$ were taken from the jugular vein and centrifuged, with serum frozen until post-vaccination samples were collected. While anesthetized, animals were tattooed with a number on the inner thigh of each leg for identification using an EZ Tatt tattoo pen (available from domestic rabbit equipment websites). Raccoons were aged based on tooth eruption charts. Nasal and oropharyngeal swab samples were taken from seventeen raccoons exhibiting either nasal or ocular discharge and sent to Cornell University Animal Health Diagnostic Center (Ithaca, NY) for detection of viral DNA using RT-PCR.

Vaccination Protocol. Raccoons were given modified-live vaccines for canine distemper, Duramune Max 5/4L (Boehringer Ingelheim Vetmedica, Inc., Fort Dodge, IA), and feline panleukopenia, Feloguard Plus3 (Boehringer Ingelheim Vetmedica, Inc., Fort Dodge, IA), at week zero, week three, and week six after arrival. Distemper and panleukopenia vaccines were given two days apart to allow for adverse reactions to be attributed to a particular vaccine. Post-vaccination blood samples were taken 14 days after the last vaccine was given and centrifuged for serum collection. Serum samples were sent to Cornell University Animal Health Diagnostic Center for titer determination. Animals then were booster vaccinated for each disease and released at 16 weeks of age.

\section{Canine Distemper Virus Neutralization}

Assay. Two-fold serum dilutions $(50 \mu \mathrm{l})$ in duplicate were mixed with 100 to 300 TCID $_{50}$ of canine distemper virus (Onderstepoort strain, Baker Institute) in a 5- $\mu 1$ volume. Mixtures were allowed to incubate for at least one hour at room temperature. A $100-\mu 1$ volume of indicator cells (Vero-ATCC) was added to each well, and plates were placed in a $\mathrm{CO}_{2}$ incubator at $37^{\circ} \mathrm{C}$ for four days. Wells were scored for the presence or absence of typical canine distemper cytopathology. Antibody values were given as titers (reciprocal of end-point dilution). Calculations used serum dilutions with a 50 percent end-point determination. 
Canine Parvovirus-2 Hemagglutination Inhibition Assay. Sera to be tested were absorbed with a 50 percent suspension of pig red blood cells to remove non-specific factors in the sera. Serial two-fold dilutions of the test sera were mixed with an equal volume of the test virus ( 4 to $8 \mathrm{HA}$ units). Canine parvovirus was used in place of feline panleukopenia due to availability and because canine parvovirus offers cross-protection antibodies against feline panleukopenia (Parrish and Carmichael 1983). Serum virus mixtures were held at room temperature for at least one hour. Pig red blood cells were added, and samples were held at $4^{\circ} \mathrm{C}$ until control antigen wells showed hemagglutination. Titers were defined as the reciprocal of the last dilution showing complete inhibition of hemagglutination.

\section{RESULTS}

Canine Distemper. PCR run on swab samples taken from the 17 animals demonstrating ocular or nasal discharge upon or shortly after entry detected 2 variants of the canine distemper virus (CDV A and CDV B). In six animals, a distemper vaccination had been given twenty-four or more hours before samples were collected, and DNA from both CDV A and B were detected in these raccoons. Because the wildtype and vaccine strains of canine distemper cannot be differentiated on PCR, the presence of the two variants in animals that had been vaccinated before sample collection was most likely due to vaccination. For animals 405, 406, and 407, however, the strong positive cycle threshold values (less than 29 used as the cut-off for strong positive reactions) may have been inconsistent with vaccination, especially for raccoon 407 , and may rather indicate natural infection. For the remaining 11 unvaccinated raccoons, CDV A was detected in 1 animal, CDV B was detected in 5 animals, and 1 animal tested positive for both variants (Table 1).

From the canine distemper prevaccination titers, five counties out of the nine from which raccoons were sampled had at least one animal that had a titer positive for exposure to the disease (greater than 1:8) (Figure 1). Thirty-one of the 49 raccoons had pre-vaccination CDV titers below levels considered protective (Pare et al 1999, found levels as low as 1:12 protective in raccoons experimentally infected). These animals all experienced increases in titer levels post-vaccination, ranging from
Table 1.Cycle threshold values of CDV A and B variants in 6 vaccinated and 11 unvaccinated juvenile raccoons using RT-PCR on samples of nasal and oropharyngeal swabs.

\begin{tabular}{|c|c|c|c|}
\hline Patient ID & $\begin{array}{c}\text { Vaccinated } \\
\text { for CDV }\end{array}$ & $\begin{array}{c}\text { Positive for } \\
\text { CDV A }\end{array}$ & $\begin{array}{c}\text { Positive for } \\
\text { CDV B }\end{array}$ \\
\hline $405^{\mathrm{a}}$ & Yes & 16.37 & 16.99 \\
\hline $406^{\mathrm{a}}$ & Yes & 15.63 & 15.76 \\
\hline $407^{\mathrm{b}}$ & Yes & 15.52 & 14.74 \\
\hline 576 & Yes & 30.44 & 29.88 \\
\hline 578 & Yes & 31.3 & 32.09 \\
\hline 647 & Yes & 32.59 & 32.99 \\
\hline 655 & No & 0 & 40.94 \\
\hline 670 & No & 0 & 0 \\
\hline 708 & No & 0 & 0 \\
\hline 714 & No & 0 & 0 \\
\hline 715 & No & 0 & 39.89 \\
\hline 716 & No & 0 & 40.25 \\
\hline 717 & No & 0 & 31.45 \\
\hline 721 & No & 0 & 35.41 \\
\hline 871 & No & 40.28 & 40.11 \\
\hline 900 & No & 40.34 & 0 \\
\hline 1009 & No & 0 & 0 \\
\hline
\end{tabular}

$a_{\text {values may be inconsistent with a vaccine. }}$

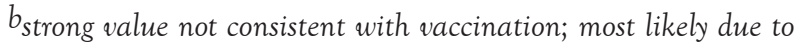
wild CDV infection.

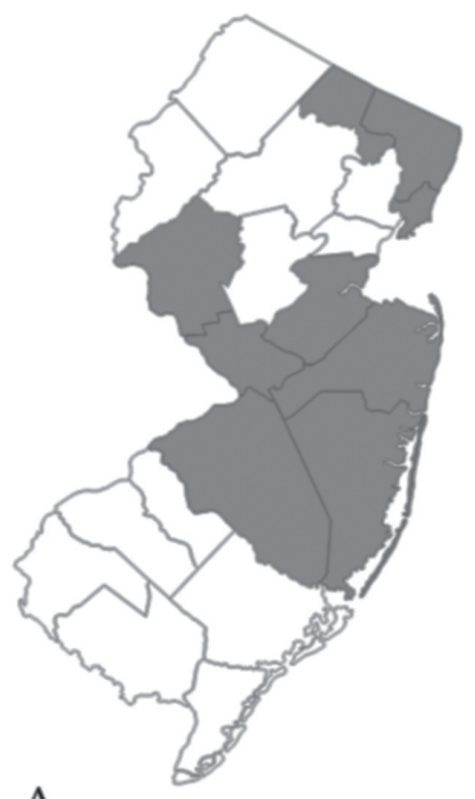

A

B

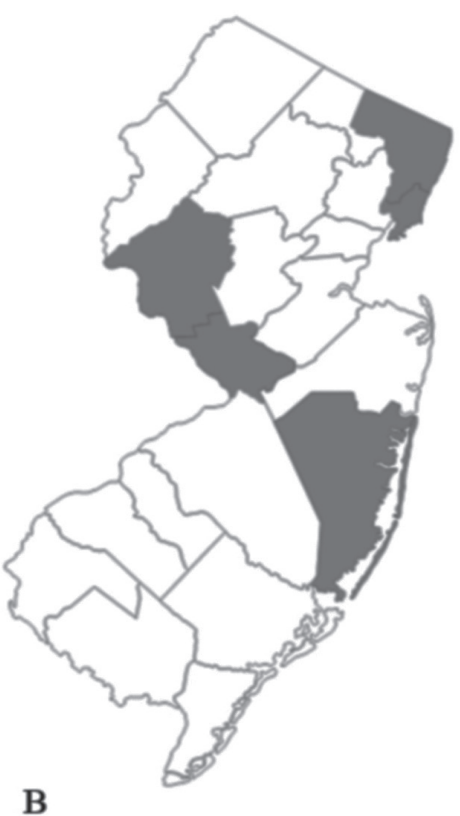

Figure 1. Map of New Jersey counties from which (A) juvenile raccoons were sampled and (B) at least one positive pre-vaccination titer (greater than 1:8) for canine distemper was obtained. 
Table 2. Pre-and post-vaccination titer levels for canine distemper in 49 juvenile raccoons following a 9-week vaccination protocol.

\begin{tabular}{|c|c|c|c|c|}
\hline Patient ID & Age Upon Entry & County & Pre-vaccination Titer & Post-vaccination Titer \\
\hline 158 & 15 days & Mercer & $<4$ & 768 \\
\hline 159 & 15 days & Mercer & $<4$ & 384 \\
\hline 214 & 12 days & Monmouth & 4 & 768 \\
\hline 215 & 12 days & Monmouth & $<4$ & 768 \\
\hline 417 & 43 days & Monmouth & $<4$ & 768 \\
\hline 457 & 43 days & Hudson & 768 & 32 \\
\hline 458 & 43 days & Hudson & 384 & 24 \\
\hline 459 & 43 days & Hudson & 768 & 48 \\
\hline 460 & 43 days & Hudson & 768 & 48 \\
\hline 461 & 43 days & Hudson & 512 & 32 \\
\hline 478 & 29 days & Monmouth & $<4$ & 768 \\
\hline 479 & 29 days & Monmouth & $<4$ & 512 \\
\hline 507 & 15 days & Mercer & $<4$ & 512 \\
\hline 543 & 33 days & Mercer & $<4$ & 768 \\
\hline 544 & 33 days & Mercer & $<4$ & 1024 \\
\hline 545 & 33 days & Mercer & $<4$ & 2048 \\
\hline 546 & 33 days & Mercer & $<4$ & 512 \\
\hline 566 & 28 days & Ocean & $<4$ & 512 \\
\hline 567 & 28 days & Ocean & $<4$ & 768 \\
\hline 568 & 28 days & Ocean & $<4$ & 768 \\
\hline 573 & 33 days & Mercer & 96 & 256 \\
\hline 575 & 33 days & Mercer & 96 & 1536 \\
\hline 577 & 33 days & Ocean & 96 & 1536 \\
\hline 579 & 33 days & Ocean & 64 & 512 \\
\hline 600 & 43 days & Middlesex & 4 & 3072 \\
\hline 601 & 43 days & Middlesex & $<4$ & 1024 \\
\hline 602 & 43 days & Middlesex & $<4$ & 384 \\
\hline 655 & 28 days & Burlington & $<4$ & 1024 \\
\hline 670 & 33 days & Hunterdon & $<4$ & 1536 \\
\hline 708 & 33 days & Hunterdon & 256 & 24 \\
\hline 714 & 49 days & Hunterdon & $<4$ & 384 \\
\hline 716 & 29 days & Hunterdon & 192 & 1024 \\
\hline 717 & 29 days & Hunterdon & 256 & 64 \\
\hline 721 & 43 days & Middlesex & $<4$ & 1536 \\
\hline 742 & 46 days & Ocean & $<4$ & 512 \\
\hline 746 & 26 days & Hunterdon & 4 & 768 \\
\hline 828 & 49 days & Mercer & $<4$ & 512 \\
\hline 871 & 43 days & Mercer & $<4$ & 1024 \\
\hline 900 & 29 days & Bergen & $<4$ & 384 \\
\hline 969 & 33 days & Bergen & $<4$ & 768 \\
\hline 970 & 54 days & Passaic & 8 & 1024 \\
\hline 973 & 49 days & Bergen & 1536 & 48 \\
\hline 974 & 49 days & Bergen & 768 & 48 \\
\hline 975 & 49 days & Bergen & 512 & 48 \\
\hline 1009 & 29 days & Mercer & $<4$ & 256 \\
\hline 1041 & 61 days & Hunterdon & *(insufficient vol.) & 2048 \\
\hline 1089 & 49 days & Mercer & 512 & 384 \\
\hline 1363 & 35 days & Middlesex & $<4$ & 1536 \\
\hline
\end{tabular}


Table 3. Pre- and post-vaccination titer levels for parvovirus in 49 juvenile raccoons following a 9-week vaccination protocol.

\begin{tabular}{|c|c|c|c|c|}
\hline Patient ID & Age Upon Entry & County & Pre-vaccination Titer & Post-vaccination Titer \\
\hline 158 & 15 days & Mercer & 20 & 640 \\
\hline 159 & 15 days & Mercer & 20 & 160 \\
\hline 214 & 12 days & Monmouth & 80 & 320 \\
\hline 215 & 12 days & Monmouth & 40 & 320 \\
\hline 417 & 43 days & Monmouth & 1280 & 40 \\
\hline 457 & 43 days & Hudson & 320 & 20 \\
\hline 458 & 43 days & Hudson & 320 & 20 \\
\hline 459 & 43 days & Hudson & 320 & 20 \\
\hline 460 & 43 days & Hudson & 640 & 20 \\
\hline 461 & 43 days & Hudson & 320 & $<20$ \\
\hline 478 & 29 days & Monmouth & 40 & 640 \\
\hline 479 & 29 days & Monmouth & 20 & 320 \\
\hline 507 & 15 days & Mercer & 160 & 20 \\
\hline 543 & 33 days & Mercer & 640 & 20 \\
\hline 544 & 33 days & Mercer & 640 & 40 \\
\hline 545 & 33 days & Mercer & 640 & 20 \\
\hline 546 & 33 days & Mercer & 640 & 20 \\
\hline 566 & 28 days & Ocean & 20 & 160 \\
\hline 567 & 28 days & Ocean & $<20$ & 640 \\
\hline 568 & 28 days & Ocean & 20 & 160 \\
\hline 573 & 33 days & Mercer & 160 & $<20$ \\
\hline 575 & 33 days & Mercer & 160 & 20 \\
\hline 577 & 33 days & Ocean & 320 & 20 \\
\hline 579 & 33 days & Ocean & 320 & $<20$ \\
\hline 600 & 43 days & Middlesex & 2560 & 80 \\
\hline 601 & 43 days & Middlesex & 5120 & 40 \\
\hline 602 & 43 days & Middlesex & 2560 & 40 \\
\hline 655 & 28 days & Burlington & 320 & $<20$ \\
\hline 670 & 33 days & Hunterdon & 1280 & 40 \\
\hline 708 & 33 days & Hunterdon & 80 & 40 \\
\hline 714 & 49 days & Hunterdon & 640 & 20 \\
\hline 716 & 29 days & Hunterdon & 40 & 40 \\
\hline 717 & 29 days & Hunterdon & 80 & 20 \\
\hline 721 & 43 days & Middlesex & 1280 & 20 \\
\hline 742 & 46 days & Ocean & $<20$ & 320 \\
\hline 746 & 26 days & Hunterdon & $<20$ & 160 \\
\hline 828 & 49 days & Mercer & 20 & 160 \\
\hline 871 & 43 days & Mercer & 5120 & 80 \\
\hline 900 & 29 days & Bergen & 640 & $<20$ \\
\hline 969 & 33 days & Bergen & $<20$ & 160 \\
\hline 970 & 54 days & Passaic & 320 & 20 \\
\hline 973 & 49 days & Bergen & 2560 & 160 \\
\hline 974 & 49 days & Bergen & 2560 & 160 \\
\hline 975 & 49 days & Bergen & 2560 & 80 \\
\hline 1009 & 29 days & Mercer & $<20$ & 40 \\
\hline 1041 & 61 days & Hunterdon & *(insufficient vol.) & 20 \\
\hline 1089 & 49 days & Mercer & 1280 & $<20$ \\
\hline 1363 & 35 days & Middlesex & 640 & $<20$ \\
\hline
\end{tabular}


$1: 384$ as the lowest response to $1: 3072$ as the greatest immune response. For animal 1041, pre-vaccination titer level could not be determined due to insufficient serum volume. The remaining 15 animals arrived with high pre-vaccination titer levels, the lowest of which being 1:64 and the highest being 1:1536. Only five of these animals demonstrated a rise in titer level after the course of vaccination, and all five had original titer levels of 1:192 or less. Titer levels decreased during the study in the other ten raccoons, although all animals had post-vaccination levels above that considered protective for the disease (Table 2). No adverse reactions to the vaccine were noted in the raccoons.

Parvovirus. All nine counties had at least one animal with a positive pre-vaccination titer (greater than 1:20) indicating exposure to parvovirus (Figure 2). Only 14 of the 49 incoming animals had pre-vaccination parvovirus titers below the level considered protective (Since no challenge studies could be found for parvovirus vaccination in raccoons, the protective titer level of 1:80 used in domestic animal medicine was chosen as the cutoff level for protection). Twelve of these animals experienced a rise in titer level postvaccination above the level needed to be protective, and responses ranged from 1:160 to 1:640. Two animals with low pre-vaccination titers did not experience appreciable rises in titers, and levels remained below those considered protective. Except for raccoon
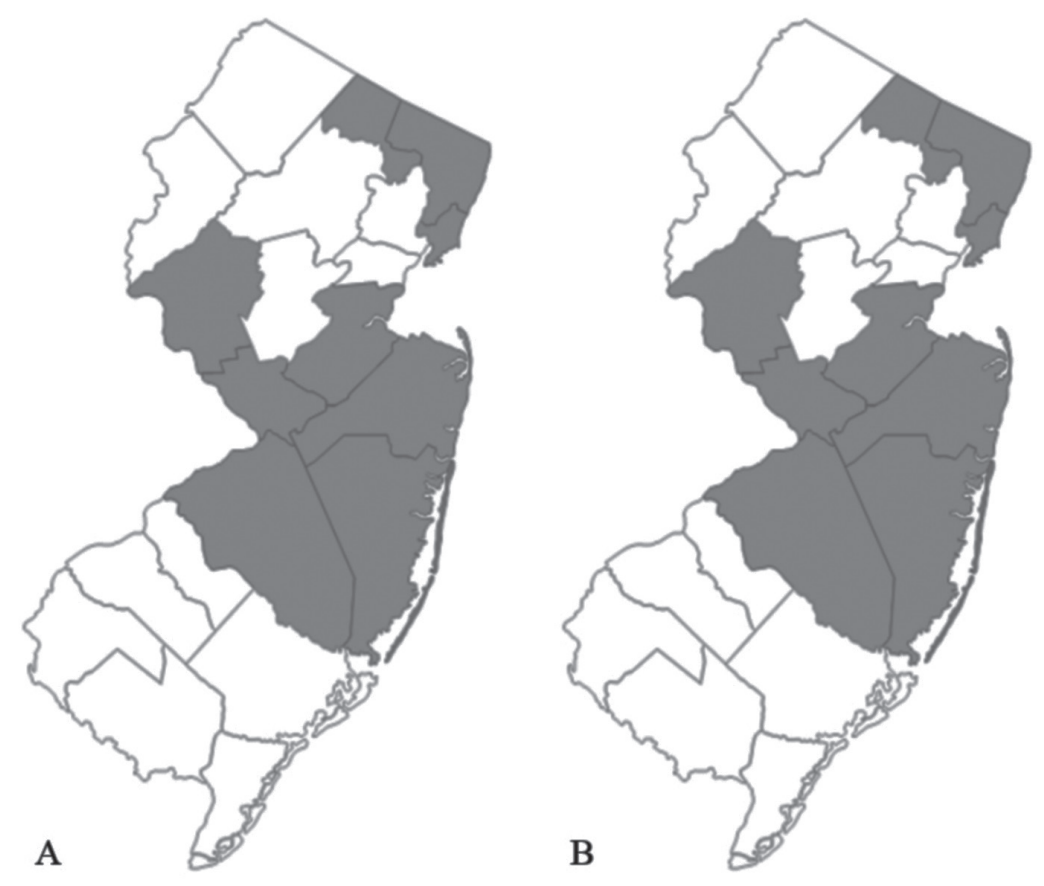

Figure 2. Map of New Jersey counties from which (A) juvenile raccoons were sampled and (B) at least one positive pre-vaccination titer (greater than 1:20) for parvovirus was obtained.
1041, for which a pre-vaccination titer could not be determined due to insufficient serum volume, the rest of the 34 animals had pre-vaccination levels from 1:80 to 1:1280. All of these animals demonstrated a decrease in titer levels over the course of the study, and only seven animals in this group had titer levels above those considered protective by the end of the study (Table 3). No adverse reactions to the vaccine were noted in the raccoons.

\section{DISCUSSION}

PCR detection of distemper virus and pre-vaccination serum titer levels in this study indicate that both canine distemper and parvovirus are present in populations of wild raccoons throughout New Jersey. The overwhelming number of counties sampled had at least one raccoon with positive pre-vaccinal titers for canine distemper and the parvoviruses, suggesting these diseases are at least epizootic in the region but are possibly enzootic, as previously proposed by Roscoe (1993).

For canine distemper, those animals that showed low pre-vaccination titers demonstrated appreciable rises in titers, well above levels considered protective for both diseases, indicating that vaccination induces an immunologic response in naïve raccoons with little to no circulating antibodies originally. Levels were high enough that it is likely immunity will last for the average lifespan of a raccoon in the wild (two to three years). All animals that showed decreases in titer levels over the course of vaccination had high pre-vaccination antibody levels upon entry, though even these levels remained above the protective cutoff. Animals with high entry levels of distemper antibodies often were littermates, suggesting the mother was exposed to the disease and maternal antibodies were passed on to the pups. Though distemper maternal antibody half-life was calculated to be 10.55 days in previous studies (Pare et al 1999), suggesting that 98 percent of circulating maternal antibodies would have been gone before the second round of vaccinations in some of these animals, colostral antibodies can last as long as 20 weeks and may have remained high enough to interfere with all three rounds of vaccination. Thus, the recommendation of finishing vaccination at 12 to 14 weeks of age used for domestic dogs (Chappius 1995) cannot be used in raccoon species if protection is to be guaranteed. 
For vaccination against the parvoviruses, animals with low pre-vaccination titers showed titer rises above protective levels in all but two cases. For these two animals, no appreciable vaccine response seemed to develop, and vaccine failure appeared to have occurred. Many more animals came in with high pre-vaccination titers for parvovirus than high titers for canine distemper, and unlike the canine distemper vaccination, for which animals with titers below 1:200 developed appreciable immune responses, only two raccoons with pre-vaccination titers above 1:20 showed post-vaccination titers above protective levels. This could indicate maternal colostral antibodies against parvoviruses last longer than those for canine distemper. Perhaps more likely, given the two incidences of vaccine failure in animals with low initial titers and the relatively low immune response to the vaccine when compared with canine distemper, the parvovirus vaccine is not as effective in eliciting an immune response in raccoons as it is in domestic species.

The evidence supporting the existence of these diseases among multiple populations of raccoons throughout the state, along with the fact no adverse vaccine reactions were observed among the 49 animals in the study, strongly supports the institution of raccoon vaccination in order to protect exotic species, domestic animals, and wildlife populations themselves. It is recommended that a standard vaccination protocol be considered for releasable rehabilitated raccoons, especially juveniles. Though wide-scale vaccination, like that of the rabies bait vaccine program, is not possible or feasible for raccoons at this time, vaccinating even a small subset of the population could be very beneficial, especially if it is targeted to reduce exposure of exotic and domestic species to unvaccinated raccoons. Though the basic reproductive rate in raccoons is not known and would vary depending on the circumstances of an outbreak, extrapolating the number from the one study that calculated $\mathrm{R}_{0}$ using red foxes (Vulpes vulpes) in Northern Italy $\left(\mathrm{R}_{0}=1.26\right)$ (Nouvette et al 2013), only around 20 percent of raccoons in a given population would need to be vaccinated for a sterilizing immunity to be achieved if the basic reproductive rate was similar. Wildlife rehabilitation centers remain in a unique position to aid in preventing the spread of these diseases by targeting for vaccination juvenile raccoons admitted. These animals, which upon losing maternal colostral antibodies become the most susceptible demographic to infection and shedding of virus, could be prevented from becoming reservoirs and strategically released to promote herd immunity. While all the concerns of using a modified-live vaccine still must be considered, including the possibility of shedding virus for a short time after vaccination, and while cost is always an issue, the overall benefit easily may outweigh the risks.

It is recommended that targeted vaccination of raccoons for canine distemper and for the parvoviruses be implemented in wildlife rehabilitation centers, as well as in areas of concern, such as zoos and wildlife sanctuaries where the trapping of raccoons is common to prevent spread of disease. A series of three vaccines induced immunity in 100 percent of naïve animals with no colostral immunity for canine distemper and 85 percent of naive animals for the parvoviruses in this study and is recommended as a minimum for use in the field. It is suggested that future wildlife vaccination protocols institute a final booster in juvenile raccoons for both distemper and parvoviruses after raccoons are twenty-weeks-old, or as close to that age as possible if animals are to be released before reaching that age, in order to promote an immune response after maternal antibodies have cleared.

\section{LITERATURE CITED}

Appel, M. J. G., et al. 1994. Canine Distemper Epizootic in Lions, Tigers, and Leopards in North America. Journal of Veterinary Diagnostic Investigation. 6(3): 277-288.

Appel, M. J. G., and B. A. Summers. 1995. Pathogenicity of Morbilliviruses for Terrestrial Carnivores. Veterinary Microbiology. 44(2-4): 187-191.

Barker, L. K., R. C. Povey, and D. R. Voigt. 1983. Response of Mink, Skunk, Red Fox and Raccoon to Inoculation with Mink Virus Enteritis, Feline Panleukopenia, and Canine Parvovirus and Prevalence of Antibody to Parvovirus in Wild Carnivores in Ontario. Canadian Journal of Comparative Medicine. 47(2): 188-197.

Bittle, J.L. 1993. Use of Vaccines in Exotic Animals. Journal of Zoo and Wildlife Medicine. 24(3): 352-356.

Chappuis, G. 1995. Control of Canine Distemper. Veterinary Microbiology. 44(2-4): 351-358.

Deem, S., et al. 2000. Canine Distemper in Terrestrial Carnivores: A Review. Journal of Zoo and Wildlife Medicine. 31(4): 441-451

Durate, M. D., et al. 2013. Snapshot of Viral Infections in Wild Carnivores Reveals Ubiquity of Parvovirus and Susceptibility of Egyptian Mongoose to Feline Panleukopenia Virus. PLoS ONE. [Cited 2013 November 15]. Available from: <http://journals.plos.org/plosone/ article?id=10.1371/journal.pone.0059399>. 
Durchfeld, B., W. Baumgartner, W. Herbst, and R. Brahm. 1990. Vaccine-associated Canine Distemper Infection in a Litter of African Hunting Dog (Lycaon pictus). Journal of Veterinary Medicine. 37(3): 203-212.

Fix, A. S., et al. 1989. Feline Panleukopenia Virus and Subsequent Canine Distemper Virus Infection in Two Snow Leopards (Panthera uncia). Journal of Zoo and Wildlife Medicine. 20(3): 273-281.

Kennedy, S., et al. 2000. Mass Die-off of Caspian Seals Caused by Canine Distemper Virus. Emerging Infectious Diseases. 6(6): 637-639.

Mochizuki, M., et al. 1996. Isolation of Canine Parvovirus from a Cat Manifesting Clinical Signs of Feline Panleukopenia. Journal of Clinical Microbiology. 34(9): 2101-2106.

Nouvette, P., et al. 2013. Rabies and Canine Distemper Virus Epidemics in the Red Fox Population of Northern Italy (2006-2010). PLoS ONE. [Cited 2014 January 1]. Available from: <http://www.plosone.org/article/info\%3Adoi\% 2F10.1371\%2Fjournal.pone. 0061588>.

Oie, K. L., et al. 1996. The Relationship Between Capsid Protein (VP2) Sequence and Pathogenicity of Aleutian Mink Disease Parvovirus (ADV): A Possible Role for Raccoons in the Transmission of ADV Infections. Journal of Virology. 70(2): 852-863.

Paddle, R. 2012. The Thylacine's Last Straw: Epidemic Disease in a Recent Mammal Extinction. Australian Zoologist. 36(1): 75-92.

Pare, J. A., et al. 1999. Humeral Response and Protection from Experimental Challenge Following Vaccination of Raccoon Pups with a Modified-live Canine Distemper Virus Vaccine. Journal of Wildlife Diseases. 35(3): 430-439.

Parrish, C. R., and L. E. Carmichael. 1983. Antigenic Structure and Variation of Canine Parvovirus Type-2, Feline Panleukopenia Virus, and Mink Enteritis Virus. Virology. 129(2): 401-414.

Parrish, C. R., C. F. Aquadro, and L. E. Carmichael. 1988. Canine Host Range and a Specific Epitope Map along with Variant Sequences in the Capsid Protein Gene of Canine Parvovirus and Related Feline, Mink, and Raccoon Parvoviruses. Virology. 166(2): 293-307.

Parrish, C. R. 1995. Pathogenesis of Feline Panleukopenia Virus and Canine Parvovirus. Baillière's Clinical Haematology. 8(1): 57-71.

Roelke-Parker, M. E., et al. 1996. A Canine Distemper Virus Epidemic in Serengeti Lions (Panthera leo). Nature. 379(6564): 441-445.
Roscoe, D. E. 1993. Epizootiology of Canine Distemper in New Jersey Raccoons. Journal of Wildlife Diseases. 29(3): 390-395.

Tratschin, J., G. K. McMaster, G. Kronauer, and G. Siegl. 1982. Canine Parvovirus: Relationship to Wild-type and Vaccine Strains of Feline Panleukopenia Virus and Mink Enteritis Virus. Journal of General Virology. 61(1): 33-41.

Truyen, U., and C. R. Parrish. 1992. Canine and Feline Host Ranges of Canine Parvovirus and Feline Panleukopenia Virus: Distinct Host Cell Tropisms of Each Virus in Vitro and in Vivo. Journal of Virology. 66(9): 5399-5408.

Van Moll, P., S. Alldinger, W. Baumgartner, and M. Adami. 1995. Distemper in Wild Carnivores: An Epidemiological, Histological, and Immunocytochemical Study. Veterinary Microbiology. 44(2-4): 193-199.

Wimsatt, J., D. E. Biggins, S. E. Williams, and V. M. Becerra. 2006. The Quest for a Safe and Effective Canine Distemper Virus Vaccine for Black-footed Ferrets. Pp. 248-266 in Recovery of the Black-Footed Ferret: Progress and Continuing Challenges, (J. E. Roelle, B. J. Miller, J. L. Godbey, and D. E. Biggins, editors). Proceedings of the Symposium on the Status of the Black-footed Ferret and Its Habitat, United States Geological Survey: Fort Collins, Colorado.

Woodroffe, R. 1999. Managing Disease Threats to Wild Mammals. The Zoological Society of London. 2(3): 185-193.

\section{ACKNOWLEDGMENTS}

This project was made possible through generous contributions from the NIH/Merial Veterinary Scholarship Program and the Morris Animal Foundation. Special thanks to the staff and volunteers of the Mercer County Wildlife Center, Michael Atchison, PhD, PI, and Kurt Hankenson, MS, DVM, $\mathrm{PhD}$, and the Cornell University Animal Health Diagnostic Center. (N

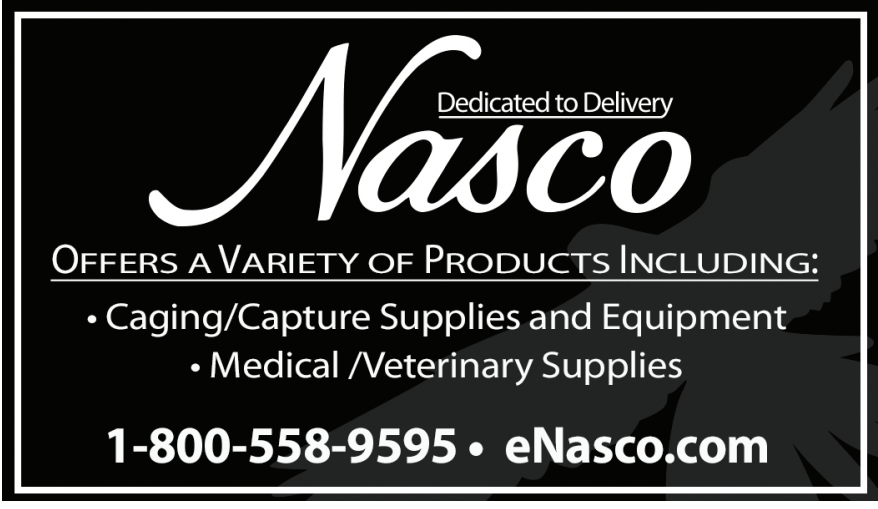

